\title{
ON SHARY'S ALGEBRAIC APPROACH FOR LINEAR INTERVAL EQUATIONS
}

\author{
ARNOLD NEUMAIER *
}

\begin{abstract}
A recent method by Shary for enclosing the solution set of a system of linear interval equations is derived in a new way. It is shown that the method converges to the fixed-point inverse, and that it has finite termination with probability 1.
\end{abstract}

Key words. linear interval equation, fixed point inverse, inner subtraction, $H$-matrix, finite termination

AMS subject classifications. $65 \mathrm{G} 10$

1. Introduction. There are a variety of stationary iterative methods for enclosing the solution set

$$
\mathbf{A}^{H} \mathbf{b}=\square\left\{x \in \mathbb{R}^{n} \mid A x=b \quad \text { for some } A \in \mathbf{A}, b \in \mathbf{b}\right\}
$$

of a system of linear equations with interval coefficient matrix $\mathbf{A} \in \mathbb{I} \mathbb{R}^{n \times n}$ and interval right hand side $\mathbf{b} \in \mathbb{I I}^{n}$. By preconditioning, the system (1) is usually reduced to another one whose coefficient matrix is an $H$-matrix.

A detailed discussions of the enclosure methods and preconditioning techniques known in 1990 is in [2], together with an analysis of the approximation power of the methods. Other recent advances concern a method by HANSEN [1], simplified and refined by Rohn [4] and Ning \& KEARFotT [3], giving optimal enclosures for problems involving an $\mathrm{H}$-matrix with diagonal midpoint, arising by midpoint preconditioning.

Recently, Shary [5] introduced a new algorithm (called by him the "algebraic approach") for enclosing the solution set (1) when $\mathbf{A}$ is an $H$-matrix. While it is an iterative method, too, he empirically observed that, in exact arithmetic, the limit is usually achieved in a finite number of iterations. In this respect, the method resembles the conjugate gradient method for linear (noninterval) equations.

In the following, we rederive Shary's method in a way that makes the finite termination property explicit. We also show that the limit interval vector of Shary's algebraic approach is the fixed point inverse of $\mathbf{A}$ applied to $\mathbf{b}$, as defined in NEUMAIER [2]. face.

In this paper, notation is as in [2], except that in interval quantities are in bold

2. A new derivation of Shary's method. Shary's algebraic method is based on the fixed point equation

$$
\mathbf{z}=\mathbf{M z}+G^{-1} \mathbf{b}
$$

where $\mathbf{M} \in \mathbb{I} \mathbb{R}^{n \times n}$ is an interval matrix, $\mathbf{b} \in \mathbb{I I}^{n}$ is an interval vector, and $G$ is a real diagonal matrix with nonzero entries. To define Shary's algorithms in a way that makes the finite termination property apparent we introduce some machinery.

*Institut für Mathematik, Universität Wien, Strudlhofgasse 4, A-1090 Wien, Austria. email: neum@cma.univie.ac.at WWW: http://solon.cma.univie.ac.at/ neum/ 
We shall need some obvious properties of the inner subtraction of two interval vectors $\mathbf{x}, \mathbf{y} \in \mathbb{I} \mathbb{R}^{n}$, defined by

$$
\mathbf{x} \stackrel{o}{\mathbf{y}}:=[\underline{x}-\underline{y}, \bar{x}-\bar{y}] .
$$

This definition also makes sense for improper interval vectors where some lower bound exceeds the corresponding upper bound.

LEMMA 2.1.

$$
\begin{gathered}
\mathbf{p}=\mathbf{x} \stackrel{\circ}{-} \mathbf{z} \Leftrightarrow \mathbf{z}=\mathbf{x} \stackrel{\circ}{-} \mathbf{p} \Leftrightarrow \mathbf{x}=\mathbf{z}+\mathbf{p} \\
(\mathbf{z}+\mathbf{q}) \stackrel{o}{-}(\mathbf{z}+\mathbf{p})=\mathbf{q}-\mathbf{p} .
\end{gathered}
$$

Proof. Insert the definitions.

Shary's concept of immersion that identifies a (possibly improper) interval vector $\mathbf{x}=[\underline{x}, \bar{x}] \in \mathbb{I I}^{n}$ with the real vector $\left(\frac{x}{\bar{x}}\right) \in \mathbb{R}^{2 n}$ can be dispensed with by defining instead the extended product

$$
\hat{B} * \mathbf{x}:=\left[B^{1} \underline{x}+B^{2} \bar{x}, B^{3} \underline{x}+B^{4} \bar{x}\right]
$$

of a real $2 n \times 2 n$-matrix

$$
\hat{B}=\left(\begin{array}{ll}
B^{1} & B^{2} \\
B^{3} & B^{4}
\end{array}\right)
$$

with four $n \times n$ blocks $B^{1}, \ldots, B^{4}$ with a (proper or improper) $n$-dimensional interval vector $\mathrm{x}=[\underline{x}, \bar{x}]$, emulating matrix vector multiplication in the immersed form.

LEMMA 2.2 .

$$
\begin{gathered}
\hat{B} *(\hat{C} * \mathbf{x})=(\hat{B} \hat{C}) * \mathbf{x} \\
\hat{B}^{-1} *(\hat{B} * \mathbf{x})=\mathbf{x} \quad \text { if } \hat{B} \text { is nonsingular } \\
\hat{B} * \mathbf{x} \stackrel{o}{-} \hat{C} * \mathbf{x}=(\hat{B}-\hat{C}) * \mathbf{x} .
\end{gathered}
$$

Proof. This follows immediately from corresponding matrix properties in dimension $2 n$.

$\square$

The importance of the extended product stems from the fact that it can be used to represent interval matrix-vector multiplication.

Proposition 2.3. For any $\mathbf{M} \in \mathbb{I I}^{n \times n}$ and $\mathbf{x} \in \mathbb{I I}^{n}$ there is a $2 n \times 2$ m matrix

$$
\hat{M}=\left(\begin{array}{cc}
M^{1} & M^{2} \\
M^{3} & M^{4}
\end{array}\right) \quad \text { with } M_{i k}^{l} \in\left\{\underline{M}_{i k}, \bar{M}_{i k}, 0\right\} \text { for all } i, k, l
$$

such that $\mathbf{M x}=\hat{M} * \mathbf{x}$. 
Proof. We have

$$
\underline{(\hat{M} \mathbf{x})_{i}}=\sum_{k=1}^{n} \underline{\mathbf{M}_{i k} \mathbf{x}_{k}}
$$

with

$$
\begin{aligned}
\underline{\mathbf{M}}_{i k} \mathbf{x}_{k} & =\min \left\{\underline{M}_{i k} \underline{x}_{4}, \underline{M}_{i k} \bar{x}_{k}, \bar{M}_{i k} \underline{x}_{k}, \bar{M}_{i k} \bar{x}_{k}\right\} \\
& =M_{i k}^{1} \underline{x}_{k}+M_{i k}^{2} \bar{x}_{k}
\end{aligned}
$$

where $\left(M_{i k}^{1}, M_{i k}^{2}\right)$ is one of $\left(\underline{M}_{i k}, 0\right),\left(0, \underline{M}_{i k}\right),\left(\bar{M}_{i k}, 0\right),\left(0, \bar{M}_{i k}\right)$, depending on which term in the min-expression is the smallest. Hence

$$
(\underline{\mathbf{M x}})_{i}=\sum_{n=1}^{n}\left(M_{i k}^{1} \underline{x}_{k}+M_{i k}^{2} \bar{x}_{k}\right)=\left(M^{1} \underline{x}+M^{2} \bar{x}\right)_{i}
$$

for all $i$, so that $\underline{\mathbf{M x}}=M^{1} \underline{x}+M^{2} \bar{x}$. By a similar argument, $\overline{\mathbf{M x}}=M^{3} \underline{x}+M^{4} \bar{x}$, where $\left(M_{i k}^{3}, M_{i k}^{4}\right)$ also takes one of the four possibilites mentioned above.

Note that $\hat{M}$ depends on $x$ and is not always unique; however, it is not difficult to extract from the proof an explicit algorithm for computing some $\hat{M}$ given $\mathbf{M}$ and $\mathbf{x}$. The fact that, independent of $\mathbf{x}$, there are only finitely many possible choices for $\hat{M}$ implies that interval matrix-vector multiplication is piecewise linear. In particular, unless $\mathbf{x}$ happens to lie on one of the hypersurfaces where the linear pieces match, $\hat{M}$ is constant in a neighborhood of $\mathbf{x}$. As we shall see, these observations explain the behavior of Shary's algorithm in practice.

We now use the extended product to give a new derivation of Shary's algorithm from which the finite termination property is apparent. Let $\mathbf{x}$ be an approximation to a solution $\mathbf{z}$ of the fixed point equation (2), and suppose that the matrix $\hat{M}$ of Proposition 2.3 satisfies both

$$
\mathbf{M} \mathbf{x}=\hat{M} * \mathbf{x} \quad \text { and } \quad \mathbf{M z}=\hat{M} * \mathbf{z} .
$$

As mentioned above, this is the generic case when $\mathbf{x}$ and $\mathbf{z}$ are sufficiently close and on the same linear piece of the multiplication operator.

THEOREM 2.4. If (10) holds and $\hat{M}-I$ is invertible then

$$
\mathbf{z}=\mathbf{x} \stackrel{o}{-}(\hat{M}-I)^{-1} *\left(\mathbf{M} \mathbf{x}+G^{-1} \mathbf{b} \stackrel{o}{-} \mathbf{x}\right) .
$$

Proof. Write $\mathbf{p}:=\mathbf{x} \stackrel{o}{\mathbf{z}}$, so that $\mathbf{x}=\mathbf{z}+\mathbf{p}$. Then

$$
\begin{array}{rlrl}
\mathbf{M} \mathbf{x}+G^{-1} \mathbf{b} \stackrel{o}{\mathbf{x}} & =\hat{M} *(\mathbf{z}+\mathbf{p})+G^{-1} \mathbf{b} \stackrel{o}{ }(\mathbf{z}+\mathbf{p}) & & \text { by }(10), \\
& =\hat{M} * \mathbf{z}+\hat{M} * \mathbf{p}+G^{-1} \mathbf{b} \stackrel{o}{ }(\mathbf{z}+\mathbf{p}) & \\
& =\mathbf{M z}+G^{-1} \mathbf{b}+\hat{M} * \mathbf{p} \stackrel{o}{ }(\mathbf{z}+\mathbf{p}) & & \text { by }(10), \\
& =(\mathbf{z}+\hat{M} * \mathbf{p}) \stackrel{o}{(\mathbf{z}+\mathbf{p})} & & \text { by }(2), \\
& =\hat{M} * \mathbf{p} \stackrel{o}{\mathbf{p}} & & \text { by }(5), \\
& =(\hat{M}-I) * \mathbf{p} & & \text { by }(9) .
\end{array}
$$

Solving for $\mathbf{p}$ using (8) gives

$$
\mathbf{p}=(\hat{M}-I)^{-1} *\left(\mathbf{M x}+G^{-1} \mathbf{b} \stackrel{o}{\mathbf{x}}\right),
$$


and since $\mathbf{x}=\mathbf{z}+\mathbf{p}$ implies $\mathbf{z}=\mathbf{x} \stackrel{o}{ } \mathbf{p}$, the assertion (11) follows.

Theorem 2.4 suggests the iteration

$$
\begin{aligned}
\mathbf{x}^{k+1}= & \mathbf{x}^{k} \stackrel{o}{ }\left(\hat{M}_{k}-I\right)^{-1} *\left(\mathbf{M x}^{k}+G^{-1} \mathbf{b} \stackrel{o}{ } \mathbf{x}^{k}\right), \\
& \text { with } \hat{M}_{k} * \mathbf{x}_{k}=\mathbf{M} \mathbf{x}_{k} \text { from Proposition 2.3. }
\end{aligned}
$$

With the initialization

$$
\mathbf{x}^{0}=(\operatorname{mid} \mathbf{A})^{-1} \mathbf{b}
$$

this is just the algebraic method (with damping factor $\tau=1$ ), as defined on p.129 of Shary [5]. In particular, Theorem 2.4 implies that as soon as $\mathbf{x}_{k}$ reaches the neighborhood of $\mathbf{z}$ that ensures (10), the method produces $\mathbf{x}^{k+1}=\mathbf{z}$ in the next step.

Shary proves convergence of his method under a technical assumption (6.1 in [5]) that is satisfied if the entries of $\mathbf{M}$ are sufficiently narrow together with Theorem 2.4 , this gives finite termination with probability 1 (i.e., unless $\mathbf{z}$ lies on two linear pieces of the multiplication operator $\mathbf{M}$ ). We also see that one cannot expect finite termination when a damping factor $\tau<1$ is used.

3. $H$-matrices and the fixed point inverse. A matrix $\mathbf{A} \in \mathbb{I} \mathbb{R}^{n \times n}$ an $H$ matrix iff the comparison matrix $\langle\mathbf{A}\rangle$ defined by

$$
\langle\mathbf{A}\rangle_{i i}=\left\langle\mathbf{A}_{i i}\right\rangle=\min \left\{|\alpha| \mid \alpha \in \mathbf{A}_{i i}\right\},
$$

$$
\langle\mathbf{A}\rangle_{i k}=-\left|\mathbf{A}_{i k}\right|=-\max \left\{|\alpha| \mid \alpha \in \mathbf{A}_{i k}\right\} \text { for } i \neq k,
$$

is nonsingular and its inverse is nonnegative, $\langle\mathbf{A}\rangle^{-1} \geq 0$. As a consequence, $0 \notin \mathbf{A}_{i i}$ for all $i$.

For $H$-matrices, the theory in Neumaier [2, Chapter 4] shows that the best enclosure that can be achieved with stationary iterations based on triangular splitting is the fixed point solution set $\mathbf{A}^{F} \mathbf{b}$, defined as the unique solution $z$ of the interval equations

$$
\mathbf{z}_{i}=\left(\mathbf{b}_{i}-\sum_{k \neq i} \mathbf{A}_{i k} \mathbf{z}_{k}\right) / \mathbf{A}_{i i} \quad(i=1, \ldots, n)
$$

(Theorem 4.4.4 in [2]). $\quad \mathbf{A}^{F} \mathbf{b}$ is computable by means of the interval Gauss-Seidel iteration, but when $\langle\mathbf{A}\rangle$ is ill-conditioned, this iteration converges very slowly. It turns out that for a suitable choice of $\mathbf{M}$ and $G$ in (2), Shary's algorithm also produces the fixed point solution set $\mathbf{z}=\mathbf{A}^{F} \mathbf{b}$, but usually much faster.

Shary defines the deviation $\operatorname{dev}(\mathbf{a})$ of an interval $\mathbf{a}=[\underline{a}, \bar{a}] \in \mathbb{I} \mathbb{R}$ from 0 to be the number

$$
\operatorname{dev}(\mathbf{a}):= \begin{cases}\underline{a} & \text { if }|\underline{a}| \geq|\bar{a}| \\ \bar{a} & \text { otherwise. }\end{cases}
$$

Using the deviation, the quotient of two intervals can be represented as the solution of a univariate fixed point equation:

Lemma 3.1. Let $\mathbf{r}, \mathbf{a} \in \mathbb{I} \mathbb{R}, 0 \in \mathbf{a}$. Then $\mathbf{z}=\mathbf{r} / \mathbf{a}$ satisfies the equation

$$
\mathbf{z}=\operatorname{dev}(\mathbf{a})^{-1}((\operatorname{dev}(\mathbf{a})-\mathbf{a}) \mathbf{z}+\mathbf{r}) .
$$


Proof. CASE 1. If $\mathbf{a}>0, \mathbf{r}>0$ then

$$
\operatorname{dev}(\mathbf{a})=\bar{a}, \quad \mathbf{z}=[\underline{r} / \bar{a}, \bar{r} / \underline{a}],
$$

and the right hand side equals

$$
\begin{aligned}
\bar{a}^{-1}([0, \bar{a}-\underline{a}][\underline{r} / \bar{a}, \bar{r} / \underline{a}]+[\underline{r}, \bar{r}]) & =\bar{a}^{-1}([0,(\bar{a}-\underline{a}) \bar{r} / \underline{a}]+[\underline{r}, \bar{r}]) \\
& =\bar{a}^{-1}[\underline{r}, \bar{a} \bar{r} / \underline{a}]=[\underline{r} / \bar{a}, \bar{r} / \underline{a}]=\mathbf{z} .
\end{aligned}
$$

CASE 2. If $\mathbf{a}>0, \mathbf{r} \ni 0$ then

$$
\operatorname{dev}(\mathbf{a})=\bar{a}, \quad \mathbf{z}=[\underline{r} / \underline{a}, \bar{r} / \underline{a}],
$$

and the right hand side equals

$$
\begin{aligned}
\bar{a}^{-1}([0, \bar{a}-\underline{a}][\underline{r} / \underline{a}, \bar{r} / \underline{a}]+[\underline{r}, \bar{r}]) & =\bar{a}^{-1}([(\bar{a}-\underline{a}) \underline{r} / \underline{a},(\bar{a}-\underline{a}) \bar{r} / \underline{a}]+[\underline{r}, \bar{r}]) \\
& =\bar{a}^{-1}[\bar{a} \underline{r} / \underline{a}, \bar{a} \bar{r} / \underline{a}]=[\underline{r} / \underline{a}, \bar{r} / \underline{a}]=\mathbf{z} .
\end{aligned}
$$

The other cases can be reduced to one of these two by changing the signs of $\mathbf{r}$ and/or a.

As explained in SHARY [5, pp. 129-130], Shary's algorithm for enclosing the solution set (1) is based on the fixed point equation (2), where

$$
G:=\operatorname{Diag}\left(\operatorname{dev}\left(\mathbf{A}_{i i}\right)\right), \quad \mathbf{M}=G^{-1}(G-\mathbf{A})
$$

and the spectral radius $\rho(|\mathbf{M}|)$ of $|\mathbf{M}|$ is assumed to be less than one. The condition $\mathbf{A}_{i i} \neq 0$ is also needed to ensure that $G$ is invertible.

(Some of Shary's theory is more general, but the only situation worked out in the algorithmic stage is the one stated here.)

TheOREM 3.2. $\mathbf{A} \in \mathbb{I I}^{n \times n}$ is an $H$-matrix iff $G$ is invertible and the spectral radius of $|\mathbf{M}|$ is less than one. In this case, $\mathbf{z}=\mathbf{A}^{F} \mathbf{b}$ is the unique solution of the fixed point equation (2).

Proof. Suppose first that $G$ is invertible and $\rho(|\mathbf{M}|)<1$. By Perron-Frobenius theory, $I-|\mathbf{M}|$ is invertible and its inverse is nonnegative. Since $G$ is diagonal and $\mathbf{A}=G(I-\mathbf{M})$ we have $\langle\mathbf{A}\rangle=\langle G\rangle(I-|\mathbf{M}|)$ and $\langle\mathbf{A}\rangle^{-1}=(I-|\mathbf{M}|)^{-1}\langle G\rangle^{-1} \geq 0$. Hence $\mathbf{A}$ is an $H$-matrix.

Conversely, suppose that $\mathbf{A}$ is an $H$-matrix. Then $0 \neq \mathbf{A}_{i i}$ whence $\operatorname{dev}\left(\mathbf{A}_{i i}\right) \neq$ 0 and $G$ is invertible. Moreover, $I-|\mathbf{M}|=\langle G\rangle^{-1}\langle\mathbf{A}\rangle$ is invertible, with inverse $(I-|\mathbf{M}|)^{-1}=\langle\mathbf{A}\rangle^{-1}\langle G\rangle \geq 0$. Again by Perron-Frobenius theory, this implies that $\rho(|\mathbf{M}|)<1$. Now let $\mathbf{z}=\mathbf{A}^{F} \mathbf{b}$. By (14) we have $\mathbf{z}_{i}=\mathbf{r}_{i} / \mathbf{A}_{i i}$, where

$$
\mathbf{r}_{i}=\mathbf{b}_{i}-\sum_{k \neq i} \mathbf{A}_{i k} \mathbf{z}_{k}
$$

Using $\operatorname{dev}\left(\mathbf{A}_{i i}\right)=G_{i i}$ we find

$$
\begin{aligned}
\mathbf{z}_{i i} & =G_{i i}^{-1}\left(\left(G_{i i}-\mathbf{A}_{i i}\right) \mathbf{z}_{i}+\mathbf{r}_{i}\right) & & \text { by Lemma } 3.1 \\
& =G_{i i}^{-1}\left(\left(G_{i i}-\mathbf{A}_{i i}\right) \mathbf{z}_{i}-\sum_{k \neq i} \mathbf{A}_{i k} \mathbf{z}_{k}+\mathbf{b}_{i}\right) & & \text { by }(18), \\
& =G_{i i}^{-1}((G-\mathbf{A}) \mathbf{z}+\mathbf{b})_{i} & & \text { since } G \text { is diagonal, } \\
& =\left(\mathbf{M z}+G^{-1} \mathbf{b}\right)_{i} & & \text { by }(17) .
\end{aligned}
$$


Hence $\mathbf{z}$ is a solution of the fixed point equation (2). Since $|\mathbf{M}|$ has spectral radius $<1$, this equation has a unique solution, so $\mathbf{z}=\mathbf{A}^{F} \mathbf{b}$ is the only solution of (2).

$\square$

Together with the good overestimation properties of $\mathbf{A}^{F} \mathbf{b}$ (derived in NEUMAIER [2]) when $\mathbf{A}$ is strictly diagonally dominant, this result explains the good numerical properties of the enclosures computed in SHARY [5].

\section{REFERENCES}

[1] E. Hansen, Bounding the solution of interval linear equations, SIAM J. Numer. Anal. 29 (1992), 1493-1503.

[2] A. Neumaier, Interval Methods for Systems of Equations, Cambridge Univ. Press, Cambridge 1990.

[3] S. Ning and R. B. Kearfott, A comparison of some methods for solving linear interval equations, SIAM J. Numer. Anal. 34 (1997), 1289-1305.

[4] J. Rohn, Cheap and tight bounds: the recent result by E. Hansen can be made more efficient, Interval Computations 4 (1993), 13-21.

[5] S.P. SHARY, Algebraic approach in the "outer problem" for interval linear equations, Reliable Computing 3 (1997), pp. 103-135. 